\title{
Copy Number Variation and Frequency of rs179008 in TLR7 Gene Associated with Systemic Lupus Erythematosus in Two Mexican Populations
}

\author{
Guillermo Valencia Pacheco $\mathbb{D},{ }^{1}$ Yumi E. Nakazawa Ueji $\mathbb{D}^{1},{ }^{1}$ Julián Ramírez Bello $\mathbb{D},^{2}$ \\ Rosa E. Barbosa Cobos $\mathbb{D}^{3}{ }^{3}$ Eduardo D. Jiménez Becerra, ${ }^{1}$ Lizbeth J. González Herrera $\mathbb{D}{ }^{4}{ }^{4}$ \\ Gerardo J. Pérez Mendoza $\mathbb{D}^{1},{ }^{1}$ Nubia A. Rivero Cárdenas, ${ }^{1}$ Angélica V. Angulo Ramírez $\mathbb{D}^{5}{ }^{5}$ \\ and Ricardo F. López Villanueva ${ }^{6}{ }^{6}$
}

\author{
${ }^{1}$ Hematology Laboratory, Regional Research Center, Autonomous University of Yucatán, Yucatán, Mexico \\ ${ }^{2}$ Endocrinology Department, National Institute of Cardiology Ignacio Chávez, México City, Mexico \\ ${ }^{3}$ Rheumatology Department, Hospital Juárez de México, México City, Mexico \\ ${ }^{4}$ Genetic Laboratory, Regional Research Center, Autonomous University of Yucatán, Yucatán, Mexico \\ ${ }^{5}$ Rheumatology Department, Hospital General Dr. Agustín O’Horán, Health Service Yucatán, Yucatán, Mexico \\ ${ }^{6}$ Rheumatology Department, Regional Hospital General (ISSSTE), Health Service Yucatán, Yucatán, Mexico
}

Correspondence should be addressed to Guillermo Valencia Pacheco; vpacheco@correo.uady.mx

Received 18 September 2021; Revised 23 November 2021; Accepted 9 December 2021; Published 17 January 2022

Academic Editor: Carlo Perricone

Copyright (c) 2022 Guillermo Valencia Pacheco et al. This is an open access article distributed under the Creative Commons Attribution License, which permits unrestricted use, distribution, and reproduction in any medium, provided the original work is properly cited.

\begin{abstract}
Systemic Lupus Erythematosus (SLE) is an autoimmune disease in which genetic factors play a role in the susceptibility to develop it. Genes related to the synthesis of interferons such as TLR7 and genetics factors such as single nucleotide polymorphisms (SNPs) or copies number variation (CNV) in the gene have been involved with the development of the disease. The genetic differences between the populations contribute to the complexity of LES. Mexico has a mestizo population with a genetic load of at least three origins: Amerindian, Caucasian, and African. The mestizo of Yucatán is the only group whose contribution Amerindian is mainly Mayan, geographically distant from other Mexican Amerindians. We analyzed the CNV and the frequency of SNP rs179008 of the TLR7 as genetic risk factors in developing the disease in patients from Yucatán and Central Mexico. Results show that $14 \%$ of the cases of the Yucatecan population showed significantly $>2 \mathrm{CNV}$ and a higher risk of developing the disease (OR: 34.364), concerning 4\% of those coming from Central Mexico (OR: 10.855). $\mathrm{T}$ allele and the A/T and T/T risk genotypes of rs 179008 were more frequent in patients of Central Mexico than in those of Yucatán (50\% vs. 30\%, 93\% vs. 30\%, $4 \%$ vs. $1 \%$ ), and association with susceptibility to develop SLE was observed (OR: 1.5 vs. $0.58,9.54$ vs. $0.66,12$ vs. 0.14 ). Data support the genetic differences between and within Mexican mestizo populations and the role of the TLR7 in the pathogenesis of SLE.
\end{abstract}

\section{Introduction}

Systemic Lupus Erythematosus (SLE) is a chronic inflammatory autoimmune disease of unknown etiology, characterized by hyperactivity of B lymphocytes and the presence of antiDNA autoantibodies, which affects women of reproductive age. It is a disease of universal distribution whose incidence and prevalence vary among populations [1-6]. In Mexico, a prevalence of $0.09 \%$ has been reported in Mexico City and $0.07 \%$ in Yucatán $[7,8]$.

Genetics play a role in the susceptibility to develop SLE, and the number of candidate genes associated with SLE has 
increased with the analysis of the human genome [9-11]; some are involved in the recognition of nucleic acids and production of interferons (IFN) $[12,13]$ while others are participating in T and B-cell signaling pathways [14, 15].

Genetic and epigenetic factors as SNPs and CNV play an essential role in autoimmunity. CNV of genes such as $C 4$, FCGR3B, CCL3L1, and TLR7 has been reported as risk factors in the development of SLE, to both susceptibilities to and to severity, as well as to other autoimmune diseases. CNV arises when a complete gene or segment of it is duplicated, having more than two copies, or when is deleted. Additional copies of these genes can promote overexpression of proteins, and its deletion leads to deficiency and functional changes [16].

Kelley et al. studied Caucasian and African American SLE patients to identify an increase in the CNV of the TLR7 and its influence on the autoantibody profile. Their results indicate that the copy number of TLR7 was variable between patients and controls, and no correlation with the phenotype of the disease, ethnic groups, and presence or absence of autoantibodies was found [17].

Garcia-Ortiz et al. investigated whether the CNV of TLR7 contributes to the development of the disease in 328 Mexican pediatric patients and 403 controls. Their results showed a significant increase in the $\mathrm{CNV}$ in female patients compared to controls and a higher association in males (OR 6.61) than in female patients (OR 3.07). Their data indicate that the increase in CNV of TLR7 may be a risk factor for developing the disease. Since TLR7 is on chromosome X and there is an increased prevalence in women, data provides evidence of an X-linked genetic component in the susceptibility and pathogenesis of SLE [18].

Approximately 267 SNPs have been found in TLR7 (GenBank Home) [19], and some are associated with the development of SLE. The SNP rs179008 (A>T) is in exon 3 of the TLR7 gene, involves the exchange of a glutamine (Gln) for a leucine (Leu) at position 11 of the amino acid sequence, and it is related to the ability of TLR7 to recognize the uracil sequences located in the single-strand RNAs (ssRNAs), thus favoring high production of IFN- $\alpha$. Gender differences based on TLR7 may influence the SLE phenotype since women have higher levels of IFN- $\alpha$ compared to men. This data suggests that increased expression of TLR7, together with increased levels of IFN- $\alpha$, contributes to the pathogenesis of SLE [20]. However, rs179008 has not been studied in the Mexican populations.

Considering the prevalence of SLE in the Mexican female population and the genetic heterogeneity among the mestizo subpopulations of Mexico, our objective was to determine the CNV and frequency of rs179008 of the TLR7 gene as genetic risk factors to develop the disease in women with SLE from Yucatán where the Amerindian contribution is mainly of Mayan ancestry [21] and from Central Mexico with different ethnical groups.

\section{Material and Methods}

2.1. Selection of Study Populations. An observational and cross-sectional study was carried out using genetic material
(DNA) stored at $-20^{\circ} \mathrm{C}$. Considering the calculations, we selected 100 samples of SLE patients and 102 healthy volunteers, all of them women of Mayan ancestry, with the inclusion criteria as individuals born in the country having a Spanish-derived last name, with Mexican Mayan ancestors back at least to the third generation, and at least one parent was born in Yucatán for two generations including their own. Women with Mayan ethnicity were selected using anthropological and demographic parameters such as language, place of birth, surnames, genealogy, and history of lifestyle to match ethnically all cases and controls. We also analyzed the samples of 151 SLE patients and 121 healthy volunteers, all of them women from the Central States of the country including Mexico City, belonging to different ethnical groups, whose samples were provided by the Department of Endocrinology, National Institute of Cardiology Ignacio Chavez, Mexico City. Patients diagnosed by a Rheumatologist, according to the criteria established by the ACR [22], signed the informed consent letter to participate in the study. Controls did not show any autoimmune or infectious disease upon entering the study and signed the informed consent letter. Pregnant women, and with other autoimmune diseases such as Rheumatoid Arthritis and Sjogren, were excluded. The Ethics Committee of Agustin O’Horán Hospital and Hospital Juárez de Mexico approved the study. The confidentiality of participants was strictly maintained.

2.2. Determination of CNV of the TLR7. DNA samples from patients and controls were quantified using Nanodrop equipment $(260$ and $280 \mathrm{~nm}$ ) to verify their concentration and purity. CNV was determined by real-time PCR using TaqMan probes marked with FAM (Applied Biosystems Hs00226289_cn). Amplification was carried out in the StepOne real-time PCR ThermoCycler (Applied Biosystems ${ }^{\circledR}$ ) using the TaqMan Universal Master Mix II reaction mixture. Thermal cycling conditions consisted of initial denaturation at $95^{\circ} \mathrm{C}$ for 10 minutes, followed by 40 cycles at $95^{\circ} \mathrm{C}$ for 15 seconds each and $60^{\circ} \mathrm{C}$ for one minute each. Three wells without genetic material and three with known DNA presented $2 \mathrm{CNV}$ of TLR7 were included as negative controls to validate the assays.

$\mathrm{CNV}$ was estimated by the $2^{\wedge \wedge \mathrm{Ct}}$ method [23], which calculates the difference in cycle thresholds (the number of PCR cycles required to produce a set of fixed thresholds) between the gene of interest and the housekeeping gene (RNAase $P$ gene, $\wedge C^{t}$ ), using the copy-caller software version 2.0@ (Applied Biosystems). Subsequent calculations normalize the $\wedge C^{t}$ of each sample to a calibrator (DNA with $2 \mathrm{CNV}$ of TLR7) that has assigned a relative expression value of 1.00 $\left(\wedge \wedge C^{t}\right)$. Assuming that the amount of PCR product doubles with each successive PCR cycle, calculating the $2-^{\wedge \wedge \mathrm{Ct}}$ value will provide the relative amount of DNA initially available for amplification in each quantitative PCR run. Therefore, the $2{ }^{\wedge} \wedge \mathrm{Ct}$ method reveals differences in the gene relative copy numbers between the samples tested. A range for each expression value was calculated based on the standard deviations of the $\wedge \wedge C^{t}$ value, where $2-\left(\left(^{\wedge C t+s}\right)\right.$ is the lower limit and $2-\left(\left(^{\wedge \mathrm{Ct}-s}\right)\right.$ is the upper limit. 


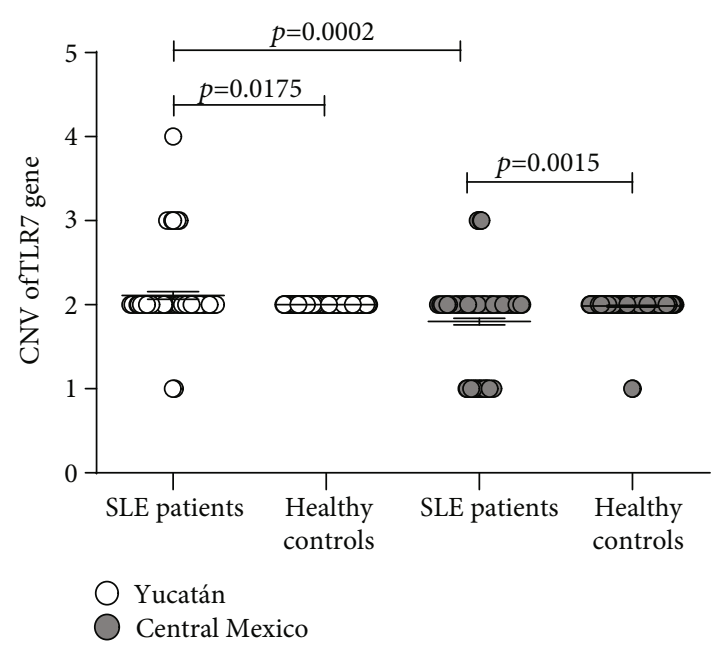

FIGURE 1: Distribution of CNV of the TLR7 between SLE patients and healthy controls from Yucatán and Central Mexico. Differences in CNV between patients and controls were analyzed as described in Material and Methods. $p$ values $<0.05$ were considered significant.

2.3. Determination of the SNP rs179008. Allelic and genotypic frequencies were determined by allelic discrimination assays with probes TaqMan (c_2259574_10). Amplification was carried out in the StepOne real-time PCR ThermoCycler (Applied Biosystems ${ }^{\circledR}$ ) using the TaqMan Universal Master Mix II reaction mixture. Thermal cycling conditions consisted of initial denaturation at $95^{\circ} \mathrm{C}$ for 10 minutes, followed by 40 cycles at $95^{\circ} \mathrm{C}$ for 15 seconds each, at $60^{\circ} \mathrm{C}$ for one minute each, and $60^{\circ} \mathrm{C}$ for 30 seconds each.

2.4. Statistical Analysis. CNV between patients and controls from both populations was analyzed employing the Wilcoxon signed-rank test of the Graph Pad Prism software. The association of CNV with SLE was determined using the MedCalc@ V19.1.7 software comparing patients and controls. To determine if the increase in the CNV is associated with the disease, patients with $>2 \mathrm{CNV}$ of Yucatán and Central Mexico were compared, as well as patients with $>2$ $\mathrm{CNV}$ and $\leq 2 \mathrm{CNV}$ with the total controls of both populations, using the EpiTools software.

Allelic and genotypic frequencies of rs 179008 were calculated with the SNPStat $\odot$ software (https://www.snpstats .net/start.htm). The association analysis was determined by comparing the genotypic and allelic frequencies between patients and controls using the MedCalc@ V19.1.7 software. The risk of susceptibility to SLE, in terms of odds ratio (OR) and confidence interval (CI 95\%), was used to determine whether alleles and genotypes of rs 179008 represent a risk factor, considering as a reference value equal to 1 . This data is null since it reflects a reason 1:1 between exposed and unexposed individuals. OR $>1$ is considered as a risk factor, and $<1$ is protective. Values of $p<0.05$ were obtained from two-tailed tests with the statistical package STATA 11.1.

The statistical power for a case-control study applicable to population genetics was estimated with the Quanto software (http://biostats.usc.edu/Quanto.html), using the fre- quency of $17 \%$ of the minor allele $\mathrm{T}$ of rs 179008 , reported in the 1000 Genomes Project Phase 3 (https://useast .ensembl.org/Homo_sapiens/Variation/Population?db= core; $\mathrm{r}=\mathrm{X}: 12885040-12886040 ; \mathrm{v}=\mathrm{rs} 179008 ; \mathrm{vdb}=$ variation; $\mathrm{vf}=$ 140870338), prevalence of SLE in Mexico [7, 8], the dominant inheritance model, the hypothesis of a single gene hypothesis, and an $\mathrm{OR}=2.5$. This calculation yielded the sample size of cases and controls of both populations to achieve $80 \%$ statistical power.

\section{Results}

3.1. CNV Analysis of TLR7 Gene. This is the first study comparing women with SLE from two Mexican populations; the Yucatecan from various municipalities of the Yucatán State with an essential contribution of Mayan ancestry, and from Central Mexico coming from the different Central States of the country (Mexico State, Guerrero, Queretaro, Oaxaca, Tlaxcala), which represent the ethnic heterogeneity in the last population.

Significant differences were found in the CNV between patients and controls and among patients, from both populations (Figure 1). We observed that $14 \%$ of Yucatecan patients showed significantly $>2 \mathrm{CNV}$ compared to $4 \%$ of those in Central Mexico and 34.36 times more at risk for developing the disease (Table 1). Patients with $>2$ CNV of Yucatán and Central Mexico were compared to determine if the increase in the CNV of the TLR7 is associated with the disease; a significant association and 3.93 times more risk of developing the disease was observed in Yucatecan. Patients with $>2$ CNV from both populations were compared with total controls, and association and risk for developing the disease were observed in them, respect at patients with $\leq 2 \mathrm{CNV}$. The data suggest that having more than two copies of TLR7 is a risk factor for developing the disease in both populations, the Yucatecan being at higher risk.

3.2. Allelic and Genotypic Frequencies of rs179008. Wild allele A was more frequent in SLE patients and healthy controls of both populations, while the risk allele $\mathrm{T}$ was less frequent in Yucatecan. However, the highest association of the $\mathrm{T}$ allele was observed in patients from Central Mexico, while in Yucatecan seems to be a protective factor (Table 2).

On the other hand, the $\mathrm{A} / \mathrm{T}$ and $\mathrm{T} / \mathrm{T}$ risk genotypes showed higher frequency and association in SLE patients from Central Mexico. Additionally, allelic and genotypic frequencies between SLE patients and controls of both populations were compared, and a significant difference between them was observed supporting the genetic differences between Mexican populations (Table 3).

\section{Discussion}

The susceptibility for developing SLE results from the interaction of multiple genes and environmental factors; however, ethnicity plays a vital role in its development, and the Amerindian population is more susceptible to developing it. Our country has a mestizo population with a genetic load of Amerindian, Caucasian, and African [24-28]. 
TABLE 1: Association analysis of CNV of the TLR7 in SLE patients and healthy controls from Yucatán and Central Mexico.

\begin{tabular}{|c|c|c|c|c|c|c|}
\hline Location & $\mathrm{CNV}$ & SLE patients $n(\%)$ & Healthy controls $n(\%)$ & OR & $95 \%$ IC & $p$ \\
\hline \multirow{2}{*}{ Yucatán } & $\leq 2$ & $86(86 \%)$ & $102(100 \%)$ & \multirow{2}{*}{34.36} & \multirow{2}{*}{$2.02-584.46$} & \multirow{2}{*}{$3.00 E-04$} \\
\hline & $>2$ & $14(14 \%)$ & $\mathrm{N} / \mathrm{D}$ & & & \\
\hline \multirow{2}{*}{ Central Mexico } & $\leq 2$ & $145(96 \%)$ & $121(100 \%)$ & \multirow{2}{*}{10.85} & \multirow{2}{*}{$0.60-194.64$} & \multirow{2}{*}{7.10 E-02 } \\
\hline & $>2$ & $6(4 \%)$ & N/D & & & \\
\hline Yucatán & $>2$ & $14(14 \%)$ & \multirow{2}{*}{-} & \multirow{2}{*}{3.93} & \multirow{2}{*}{$1.45-10.61$} & \multirow{2}{*}{$8.40 E-03$} \\
\hline Central Mexico & $>2$ & $6(4 \%)$ & & & & \\
\hline Yucatán/Central Mexico & $>2$ & $14+6(14 \%+4 \%)$ & $102+121(100 \%+100 \%)$ & 2.76 & $1.02-7.46$ & $4.40 E-03$ \\
\hline Yucatán/Central Mexico & $\leq 2$ & $86+145(86 \%+96 \%)$ & $102+121(100 \%+100 \%)$ & 0.70 & $0.48-1.02$ & $6.61 E-02$ \\
\hline
\end{tabular}

N/D: no detected.

TABLE 2: Association analysis of the allelic and genotypic frequencies of rs179008 (A>T) in SLE patients and healthy controls from Yucatán and Central Mexico.

\begin{tabular}{|c|c|c|c|c|c|c|c|c|}
\hline Location & Allele and genotype & SLE patients & HW & Healthy controls & HW & OR & $95 \%$ IC & $p$ \\
\hline \multirow{5}{*}{ Yucatán } & $\mathrm{A}$ & $168(84 \%)$ & \multirow{5}{*}{0.45} & $154(75 \%)$ & & \multicolumn{3}{|c|}{ Reference } \\
\hline & $\mathrm{T}$ & $32(16 \%)$ & & $50(25 \%)$ & & 0.58 & $0.3577-0.9620$ & $3.4 E-02$ \\
\hline & $\mathrm{A} / \mathrm{A}$ & $69(69 \%)$ & & $58(57 \%)$ & 1 & \multicolumn{3}{|c|}{ Reference } \\
\hline & $\mathrm{A} / \mathrm{T}$ & $30(30 \%)$ & & $38(37 \%)$ & & 0.66 & $0.3669-1.200$ & $1.7 E-01$ \\
\hline & $\mathrm{T} / \mathrm{T}$ & $1(1 \%)$ & & $6(6 \%)$ & & 0.14 & $0.0163-1.1974$ & $7.3 E-02$ \\
\hline \multirow{5}{*}{ Central Mexico } & $\mathrm{A}$ & $152(50 \%)$ & \multirow{5}{*}{$<0.0001$} & $148(61 \%)$ & & \multicolumn{3}{|c|}{ Reference } \\
\hline & $\mathrm{T}$ & $150(50 \%)$ & & $94(39 \%)$ & & 1.55 & $1.1025-2.1896$ & $1.2 E-02$ \\
\hline & $\mathrm{A} / \mathrm{A}$ & $5(3 \%)$ & & $30(25 \%)$ & $<0.0001$ & \multicolumn{3}{|c|}{ Reference } \\
\hline & $\mathrm{A} / \mathrm{T}$ & $140(93 \%)$ & & $88(73 \%)$ & & 9.54 & $3.5696-25.5252$ & $<1.0 E-04$ \\
\hline & $\mathrm{T} / \mathrm{T}$ & $6(4 \%)$ & & $3(2 \%)$ & & 12 & $2.2400-64.2865$ & $3.7 E-03$ \\
\hline
\end{tabular}

TABLE 3: Comparison of allelic and genotypic frequencies of the rs179008 between SLE patients and healthy controls from Yucatán and Central México.

\begin{tabular}{lcc}
\hline rs179008 & $\begin{array}{c}\text { SLE patients Yucatán vs. } \\
\text { SLE patients Central } \\
\text { México }\end{array}$ & $\begin{array}{c}\text { Healthy controls Yucatán vs. } \\
\text { healthy controls Central } \\
\text { México }\end{array}$ \\
\hline $\mathrm{A} / \mathrm{A}$ & $p=<1.00 E-07$ & $p=1.00 E-06$ \\
$\mathrm{~A} / \mathrm{T}$ & & \\
$\mathrm{T} / \mathrm{T}$ & & $p=1.20 E-03$ \\
$\mathrm{~A}$ & $p=<1.00 E-07$ & \\
$\mathrm{~T}$ & & \\
\hline
\end{tabular}

Silva-Zolezzi et al. evaluated the genetic diversity and ancestry of 600 Mexican mestizos proceeding from Sonora, Zacatecas, Guanajuato, Guerrero, Veracruz, and Yucatán, and they observed genetic differences between and within Mexican mestizo populations. The subpopulation of Yucatán is the only mestizos with an Amerindian ancestral component, mainly Maya, and represents an ethnic group geographically distant from other Mexican Amerindian groups [29]. This characteristic could be influencing the development of SLE, compared to the population of Central Mexico, supporting the importance of studying the CNV of genes involved in the disease.
Kelley et al. observed differences in the CNV of the TLR7 in patients and controls Caucasian and African Americans but no association as a genetic risk factor for the development of SLE, which contrast with our results [17]. On the contrary, our data seem to correlate with Garcia-Ortiz et al. whose observed association $(\mathrm{OR}=3.07, p<1.00 E-$ 04) of CNV of the TLR7 with the disease in the female child population of Central Mexico [18]. Their results also support what we previously reported: $>2 \mathrm{CNV}$ of the TLR7 in women of Mayan descent [30]. Data suggest that the CNV of the TLR7 is a risk factor for developing the disease in Yucatecan and Central Mexico women. Gender stratification was not possible since all patients were female; however, it is crucial to consider the participation of male patients in subsequent studies. On the other hand, having more or less than two copies of the TLR7 does not mean that the person develops or presents the disease since it depends on the combination of genetic, environmental, and hormonal factors to trigger it.

There are few association studies of rs179008 (Table 4). Sánchez et al. found a higher frequency of the risk allele (T) of rs179008 in SLE females from the Spanish Caucasian population but was not associated with the development of the disease [31]. dos Santos et al. analyzed the allelic and genotypic frequencies of rs179008 in Brazilian female patients and observed that it might be a susceptibility factor 
TABLE 4: Studies on rs179008 of TLR7 associated with SLE in Mexico and other populations.

\begin{tabular}{cccccc}
\hline & Population & SLE patients/control & OR $(95 \%$ CI $)$ & $p$ & Ref. \\
\hline & Yucatán/Central Mexico & $251 / 223$ & $1.53(1.102-2.189)$ & $1.20 E-02$ & ${ }^{*}$ \\
rs179008 & Spanish Caucasian & $752 / 1107$ & $0.97(0.80-1.54)$ & $7.00 E-01$ & {$[31]$} \\
& Brazilian & $370 / 415$ & $1.74(1.2-2.70)$ & $3.00 E-03$ & {$[32]$} \\
& Danish & $142 / 443$ & NA & US & {$[33]$} \\
& African & $11,984 / 14,572$ & $0.43(0.238-0.775)$ & $5.00 E-03$ & {$[34]$} \\
\hline
\end{tabular}

NA: no association reported; US: unreported significance. ${ }^{*}$ Current study.

to develop the disease [32]. Enevold et al. found no association of these polymorphisms with SLE in patients from the Danish population [33]. Lee et al. conducted a metaanalysis of the relationship between 12 TLR polymorphisms and SLE susceptibility. The authors included 26 studies that involved 11,984 patients and 14,572 controls and observed an association of rs179008 and SLE in African but not in the Caucasian population [34].

This is the first report of rs179008 in two Mexican populations, and a significant association of the $\mathrm{T}$ allele, and $\mathrm{A} / \mathrm{T}$ and $\mathrm{T} / \mathrm{T}$ genotypes, was observed in Central Mexico women and suggests that it is a risk factor for them to develop the disease but not in Yucatecan women. These results may be influenced by genetic differences between Mexican populations, supporting the participation of the ancestral component. It is convenient to emphasize that the Yucatecan patients constitute a population of Mayan women, without ancestral substructure and history until the third generation in Yucatán, unlike those patients in Central Mexico [21]. Furthermore, we cannot exclude other SNPs of the TLR7 that may be contributing to the development of SLE since the rs179008 is in a region of known CNV, and alleles may differ with the number of copies.

The sample size with sufficient statistical power is critical to accomplish the genetic risk variant association in human complex diseases such as SLE. The statistical power of $80 \%$ is to avoid false-negative associations and determine a cost-effective sample size in large-scale association studies. Analysis testing a single SNP requires 248 cases while screening 500,000 SNPs requires 1,206, based on the following criteria: an OR of $2.5 \%$ disease prevalence, 5\% minor allele frequency, 1:1 case/control ratio, and 5\% error rate in an allelic test [35]. Analyzing a single SNP under a dominant model and an OR of 2.5, the sample size of our study has enough power $(>80 \%)$ to detect the effect of the rs179008.

\section{Conclusion}

The results support the role of the TLR7 in the pathogenesis of SLE in Mexican mestizo and suggest that extra copies of the gene may be a risk factor for developing the disease in the Mayan population. The presence of the T allele, and A/ $\mathrm{T}$ and $\mathrm{T} / \mathrm{T}$ genotypes of rs 179008 , associated with the disease in patients from Central Mexico, supports the genetic differences between and within Mexican mestizo populations with different ethnical backgrounds.

\section{Data Availability}

The SNP rs179008 data of TLR7 gene, as well as softwares used to support the findings of this study, are included within the article.

\section{Conflicts of Interest}

The authors declare that there is no conflict of interest regarding the publication of this manuscript.

\section{Acknowledgments}

The work was supported by the CONACYT (National Council of Science and Technology), grant FONSECSALUD, 2010-1-139788.

\section{References}

[1] J. A. Mills, "Systemic lupus erythematosus," The New England Journal of Medicine., vol. 330, no. 26, pp. 1871-1879, 1994.

[2] T. J. Vyse and B. L. Kotzin, "Genetic susceptibility to systemic lupus erythematosus," Annual Review of Immunology, vol. 16, no. 1, pp. 261-292, 1998.

[3] P. E. Lispky, "Systemic lupus erythematosus: an autoimmune disease of B cell hyperactivity," Nature Immunology, vol. 2, pp. 764-766, 2001.

[4] A. H. Sawalha and J. B. Harley, "Antinuclear autoantibodies in systemic lupus erythematosus," Current Opinion in Rheumatology, vol. 16, no. 5, pp. 534-540, 2004.

[5] K. Yasumoto, "Pathological lymphocyte activation by defective clearance of self-ligands in systemic lupus erythematosus," Rheumatology, vol. 42, no. 2, pp. 214-222, 2003.

[6] R. Lahita, "The role of sex hormone in systemic lupus erythematosus," Current Opinion in Rheumatology, vol. 11, no. 5, pp. 352-356, 1999.

[7] I. Pelaez-Ballestas, L. H. Sanin, J. Moreno-Montoya et al., "Epidemiology of the rheumatic diseases in Mexico. A Study of 5 regions based on the COPCORD methodology," The Journal of Rheumatology. Supplement, vol. 86, pp. 3-8, 2011.

[8] J. Alvarez-Nemegyei, I. Pelaez-Ballestas, L. H. Sanin, M. H. Cardiel, A. RAMIREZ-ANGULO, and M. V. GOYCOCHEA-ROBLES, "Prevalence of musculoskeletal pain and rheumatic diseases in the southeastern region of Mexico. A COPCORD-Based community survey," The Journal of Rheumatology. Supplement, vol. 86, pp. 21-25, 2011.

[9] International Consortium for Systemic Lupus Erythematosus Genetics (SLEGEN), J. B. Harley, M. E. Alarcón-Riquelme et al., "Genome-wide association scan in women with systemic lupus erythematosus identifies susceptibility variants in 
ITGAM, PXK, KIAA1542 and other loci," Nature Genetics, vol. 40, no. 2, pp. 204-210, 2008.

[10] S. V. Kozyrev, A. K. Abelson, J. Wojcik et al., "Functional variants in the B-cell gene_BANK1_are associated with systemic lupus erythematosus," Nature Genetics, vol. 40, no. 2, pp. 211216, 2008.

[11] G. Hom, R. R. Graham, B. Modrek et al., "Association of systemic lupus erythematosus withC8orf13-BLKandITGAMITGAX," The New England Journal of Medicine., vol. 358, no. 9, pp. 900-909, 2008.

[12] R. R. Graham, C. Kyogoku, S. Sigurdsson et al., "Three functional variants of IFN regulatory factor 5 (IRF5) define risk and protective haplotypes for human lupus," Proceedings of the National Academy of Sciences of the United States of America, vol. 104, no. 16, pp. 6758-6763, 2007.

[13] A. K. Abelson, A. M. Delgado-Vega, S. V. Kozyrev et al., "STAT4 associates with systemic lupus erythematosus through two independent effects that correlate with gene expression and act additively with IRF5 to increase risk," Annals of the Rheumatic Diseases, vol. 68, no. 11, pp. 1746-1753, 2009.

[14] D. S. C. Graham, R. R. Graham, H. Manku et al., "Polymorphism at the TNF superfamily gene_TNFSF4_confers susceptibility to systemic lupus erythematosus," Nature Genetics, vol. 40, no. 1, pp. 83-89, 2008.

[15] R. Lu, G. S. Vidal, J. A. Kelly et al., "Genetic associations of LYN with systemic lupus erythematosus," Genes and Immunity, vol. 10, no. 5, pp. 397-403, 2009.

[16] T. Ptacek, X. Li, J. Kelley, and L. Edberg, "Copy number variants in genetic susceptibility and severity of systemic lupus erythematosus," Cytogenetic and Genome Research, vol. 123, pp. 142-147, 2008.

[17] J. Kelley, M. R. Johnson, G. S. Alarcon, R. P. Kimberly, and J. C. Edberg, "Variation in the relative copy number of the TLR7 gene in patients with systemic lupus erythematosus and healthy control subjects," Arthritis and Rheumatism, vol. 56, no. 10, pp. 3375-3378, 2007.

[18] H. Garcia-Ortiz, R. Velazquez-Cruz, F. Espinosa-Rosales, S. Jimenez-Morales, V. Baca, and L. Orozco, "Association of TLR7 copy number variation with susceptibility to childhood-onset systemic lupus erythematosus in Mexican population," Annals of the Rheumatic Diseases, vol. 69, no. 10, pp. 1861-1865, 2010.

[19] GenBank Home, “GeneCards," 2021, http://www.genecards .org/cgi-bin/carddisp.pl?gene=TLR7\&snp=267\&rf=/home/ genecards/current/website/carddisp.pl\#snp.

[20] B. Berghöfer, T. Frommer, G. Haley, L. Fink, G. Bein, and H. Hackstein, "TLR7 ligands induce higher IFN- $\alpha$ production in females," Journal of Immunology, vol. 177, pp. 2088-2096, 2006.

[21] L. González-Herrera, J. E. Sosa-Escalante, P. López-González et al., "Ancestral proportions based on 22 autosomal STRs of an admixed population (Mestizos) from the Peninsula of Yucatán, Mexico," Forensic Science International: Genetics Supplememt Series, vol. 7, no. 1, pp. 429-431, 2019.

[22] C. Bombardier, D. D. Gladman, M. B. Urowitz, D. Caron, and C. H. Chang, "Derivation of the SLEDAI. A disease activity index for lupus patients. The committee on prognosis studies in SLE," Arthritis and Rheumatism, vol. 35, pp. 630-640, 1992.

[23] A. Giulietti, L. Overbergh, D. Valckx, B. Decallonne, R. Bouillon, and C. Mathieu, "An overview of real-time quan- titative PCR: applications to quantify cytokine gene expression," Methods, vol. 25, no. 4, pp. 386-401, 2001.

[24] N. Danchenko, J. Satia, and M. Anthony, "Epidemiology of systemic lupus erythematosus: a comparison of worldwide disease burden," Lupus, vol. 15, pp. 308-318, 2006.

[25] M. E. Alarcón-Riquelme, J. T. Ziegler, J. Molineros et al., "Genome-wide association study in an amerindian ancestry population reveals novel systemic lupus erythematosus risk loci and the role of european admixture," Arthritis and Rheumatism, vol. 68, no. 4, pp. 932-943, 2016.

[26] R. Rubí Castellanos, G. Martínez Cortes, J. F. Muñoz Valle et al., "Pre-hispanic mesoamerican demography approximates the present-day ancestry of mestizos throughout the territory of Mexico," American Journal of Physical Anthropology, vol. 139, pp. 284-285, 2009.

[27] G. Martínez-Cortés, J. Salazar-Flores, J. Haro-Guerrero et al., "Maternal admixture and population structure in MexicanMestizos based on mtDNA haplogroups," American Journal of Physical Anthropology, vol. 151, no. 4, pp. 526-537, 2013.

[28] N. Yang, H. Li, L. Criswell et al., "Examination of ancestry and ethnic affiliation using highly informative diallelic DNA markers: application to diverse and admixed populations and implications for clinical epidemiology and forensic medicine," Human Genetics, vol. 118, no. 3-4, pp. 382-392, 2005.

[29] I. Silva-Zolezzi, A. Hidalgo-Miranda, J. Estrada-Gil et al., "Analysis of genomic diversity in Mexican Mestizo populations to develop genomic medicine in Mexico," Proceedings of the National Academy of Sciences of the United States of America, vol. 106, no. 21, pp. 8611-8616, 2009.

[30] G. V. Pacheco, D. C. Cruz, L. J. González Herrera et al., "Copy number variation of TLR-7 gene and its association with the development of systemic lupus erythematosus in female patients from Yucatán Mexico," Genetics \& Epigenetics, vol. 6, pp. 31-36, 2014.

[31] E. Sánchez, J. L. Callejas-Rubio, J. M. Sabio et al., "Investigation of TLR5 and TLR7 as candidate genes for susceptibility to systemic lupus erythematosus," Clinical and Experimental Rheumatology, vol. 27, no. 2, pp. 267-271, 2009.

[32] B. dos Santos, J. Valverde, P. Rohr et al., “TLR7/8/9 polymorphisms and their associations in systemic lupus erythematosus patients from Southern Brazil," Lupus, vol. 21, no. 3, pp. 302309, 2012.

[33] C. Enevold, C. H. Nielsen, R. S. Jacobsen et al., "Single nucleotide polymorphisms in genes encoding toll-like receptors 7,8 and 9 in Danish patients with systemic lupus erythematosus," Molecular Biology Reports, vol. 41, no. 9, pp. 5755-5763, 2014.

[34] Y. H. Lee, S. J. Choi, J. D. Ji, and G. G. Song, “Association between toll-like receptor polymorphisms and systemic lupus erythematosus: a meta-analysis update," Lupus, vol. 25, no. 6, pp. 593-601, 2016.

[35] E. P. Hong and J. W. Park, "Sample size and statistical power calculation in genetic association studies," Genomics \& Informatics., vol. 10, no. 2, pp. 117-122, 2012. 